\title{
Simulation Research on Vehicle Signal in Vehicle Axle Load Monitoring System Based on LabVIEW
}

\author{
Weiguo Di ${ }^{1, \text { a }}$, Ming Yang ${ }^{2, b}$, Yan Yan ${ }^{3, c}$ \\ ${ }^{1,2,3}$ School of Electrical and Electronics Engineering,Shijiazhuang Tiedao University, Shijiazhuang, \\ 050043, China \\ agwd77@126.com, byangming9915@126.com, cyanyanhello@126.com
}

Keywords: axle load; simulation; vehicle signal; LabVIEW

Abstract. Through analyzing vehicle axle load monitoring system and drawing on vehicle monitoring principle which uses geomagnetic coils and piezoelectric sensors, the simulation of vehicle signal is achieved. In the course of simulation LabVIEW and PCI-6013 data acquisition card are used. The editing and measurement of axle load monitoring systems can be achieved by using the vehicle simulation signal in the laboratory.

\section{Introduction}

With the development of the road transportation and commerce the phenomena of vehicle overload are increasing on the road, and vehicle dynamic weighing technology has become a key technology and direction for measuring vehicle loads [1,2]. In this paper, the existing vehicle signal of axle load monitoring system is simulated, the test of the vehicle signal monitoring system can be easily carried in the laboratory, and the risk and inefficient of outside work are avoid.

\section{Principle of axle load measurement}

The monitoring of vehicles is completed by the use of the geomagnetic coils and the piezoelectric sensors [3]. The geomagnetic coils can produce the conversion of high and low level according to the change of magnetic induction. When the vehicle is near, the high level will transform to the low level. The piezoelectric sensor will produce voltage change by the change of pressure, which can detect axle load, axle speed, wheelbase of the vehicle[4]. The installation of geomagnetic coils and piezoelectric sensors is shown in Fig. 1. The geomagnetic coil can induct the vehicle when the vehicle entrys into the detection zone. Then the output signal changes from high level to low level. When the program detects the change peak value judgment is beginning. And the records of the number, position and appearing time of peak value are achieved through the methods of curve fitting and peak value judgment. It shows that the vehicle leaves detection area when the rising edge signal of another geomagnetic coil is detected. Curve fitting method can be achieved by sub-VI in LabVIEW, and the necessary requirements are achieved by setting the fitting accuracy; Peak value judgment method can also be achieved by sub-VI, and peak detection can be realized by setting the width of peak value.

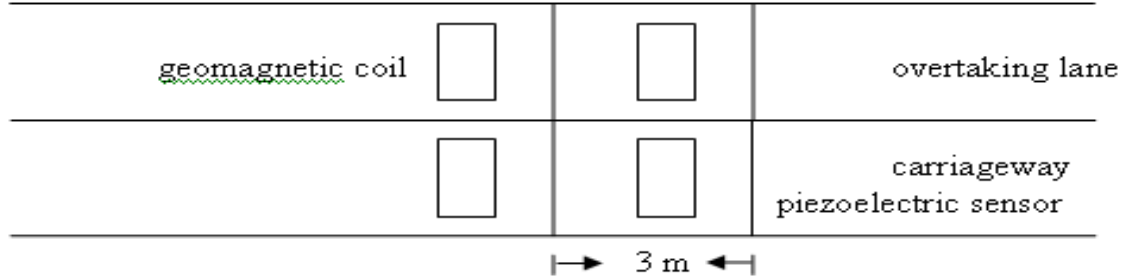

Fig. 1 Installation of geomagnetic coils and piezoelectric sensors

The further analysis need to conduct after the information of vehicle is collected. The axle load information is determined by the width of peak value. There are two ways: one is to calculate and display the information of axle load by using the integral method of from the beginning of peak value to the end of the peak value. The other is to add the values above the threshold peak value together by using the method of peak value judgment. The axle speed can be separated by two piezoelectric sensors 
fixed three meters, and then we can calculate the axle speed by detecting the time difference of peak value. Wheelbase can be calculated by the axle speed and the peak time [5].

\section{Axle load measurement software design process}

Axle load measurement software is to achieve the accurate axle load information based on the geomagnetic coil and the piezoelectric sensor. The geomagnetic coil provides early warning information of vehicle arriving. The piezoelectric sensor judges the peak value and analyzes the corresponding axle load information, and the axle speed and wheelbase are calculated depend on the information of the piezoelectric sensor.

According to the principle of axle load monitoring system the vehicle signal is simulated through the signal simulation of piezoelectric sensors and magnetic coil. And the random numbers are used to simulate the randomness of the vehicles on the highway.

\section{Software design of simulation signal in vehicle monitoring system}

The modular design is adopted, the vehicle signal generating system is divided into several functional modules, such as piezoelectric sensor simulation signal module, geomagnetic coil simulation signal module, data acquisition module, data storage and display module. The roles of every module are as follows:

(1) Piezoelectric sensor simulation signal: the piezoelectric sensors simulation signal is used to determine axle load, shaft speed, wheelbase and other information of the vehicle signal.

(2) Geomagnetic coil simulation signal: the geomagnetic coil simulation signal is used to determine whether a vehicle reached and assist to divide the vehicle.

(3) Data acquisition module: the PCI-6013 output port signals are collected and displayed on the front panel.

(4) Data storage and display: the output binary informations are displayed and stored in the appointed file.

The above four modules compose the vehicle signal simulation program, and achieve the simulation of vehicle signal. The following will makes a concrete analysis of the specific function, principle and implementation program of each part[6].

Simulation of the piezoelectric sensor signal. Axle load sensor uses Roadtrax BL piezoelectric film traffic sensor which is produced by the Measurement Specialties company in America. It will produce the charge signal which is proportional to the force when the piezoelectric material is under external force. The counter port of PCI-6013 data acquisition card simulates piezoelectric sensor signal, the square wave signal is generated by controlling the change of output pulse of counter port, then the vehicle axle load is simulated by controlling the width of the square wave signal, its simulation principle is that the axle speed and wheelbase of the vehicle signal are simulated by controlling the peak location of square wave pulse. The program of piezoelectric sensor simulation signal is based on the generation program of square wave signal, through different combinations to generate the square wave of simulated vehicle signal. The low pulse width of square wave is controled by delay, high pulse is outputed from the counter. Pulse signal generation program is shown in Fig. 2. Pulse signal generation program is the basis of the piezoelectric sensor simulation signal program. For the convenience of applications it is edited to the sub-VI which is applied in the program. The pulse signal sub-VIs form vehicle signal through the combination, and they can form two axles vehicle, three axles vehicle, four axles vehicle, five axles vehicle, six axles vehicle and other vehicles by the different combination. Here the program block diagram of only two axles vehicle and six axles vehicle are gived. The program block diagram of two axles vehicle is shown in Fig. 3. The program block diagram of six-axle vehicle is shown in Fig. 4. 


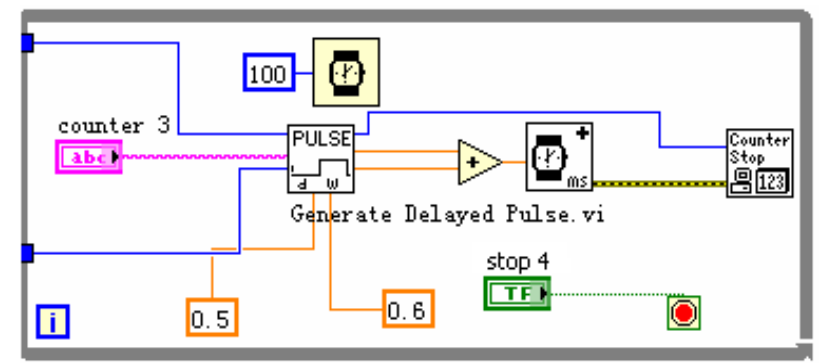

Fig. 2 Pulse signal generation program

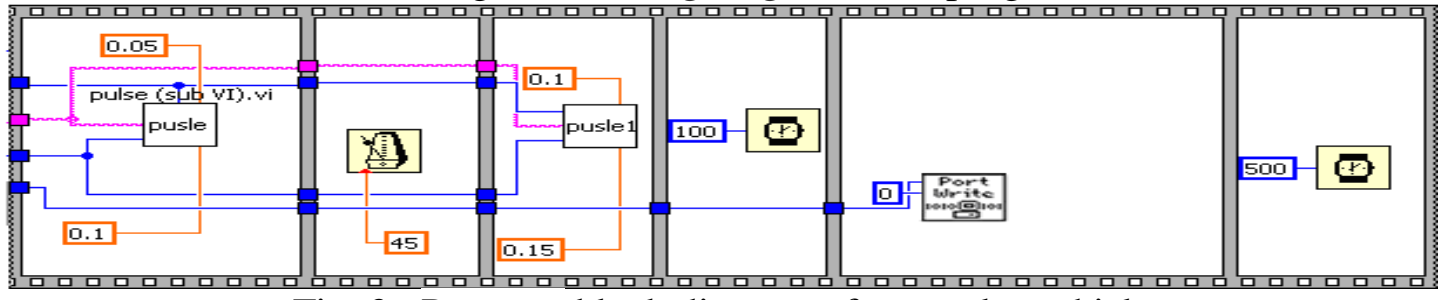

Fig. 3 Program block diagram of two axles vehicle

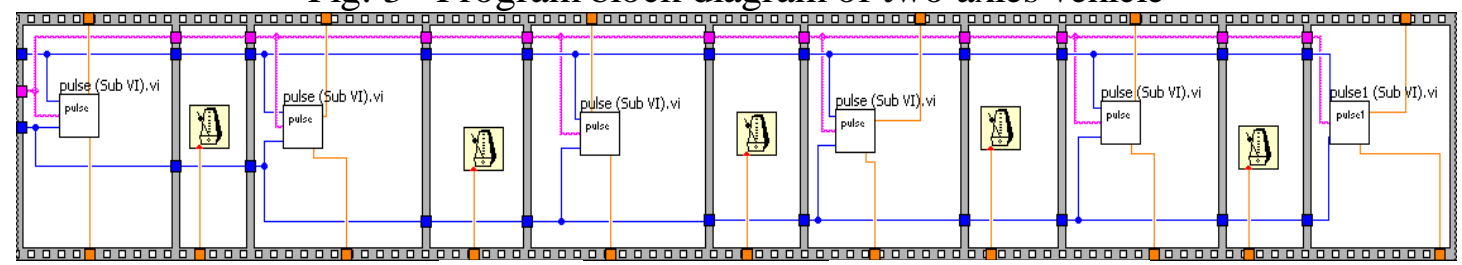

Fig. 4 Program block diagram of six axles vehicle

Simulation of geomagnetic coil signal. The simulation of geomagnetic coil signal is achieved by the digital port of PCI-6013, the LabVIEW virtual instrument development platform is used, the high and low levels of the digital output port are controled by port write, and the simulation of geomagnetic coil signal is achieved. The program block diagram of geomagnetic coil signal is shown in Fig. 5.

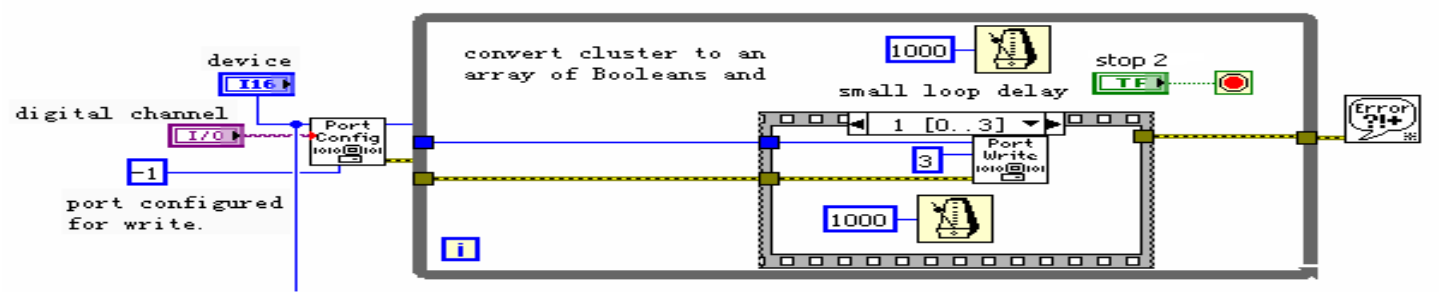

Fig. 5 Program block diagram of geomagnetic coil signal

Data acquisition module.PCI-6013 can provide economical and reliable data acquisition function. The signals are collected in order to achieve accurate simulation of the vehicle signal, so that they can contrast with the field acquisition signals, and easy to edit and adjust the programs. The analog input ports of the data acquisition card are applied to collect timer output ports and digital output ports, thus the simulation of vehicle signal is achieved. The program block diagram of data acquisition module is shown in Fig. 6.

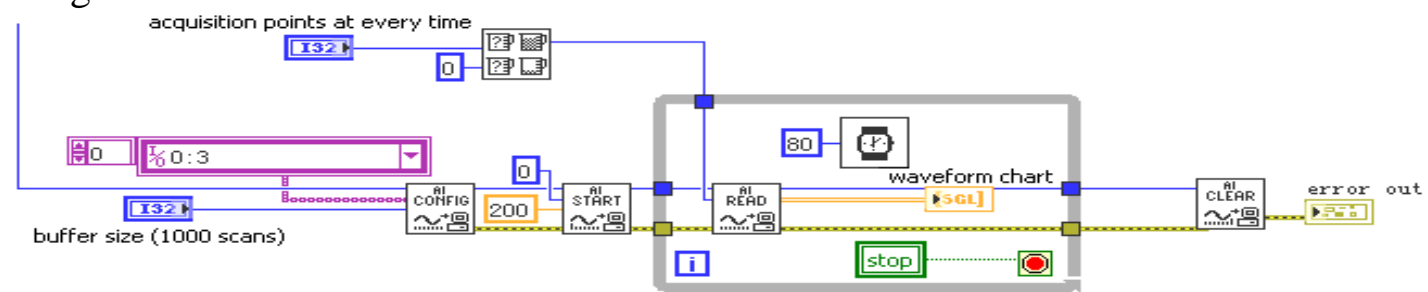

Fig. 6 Program block diagram of data acquisition module

Data storage and display. The spreadsheet is used in data display. Spreadsheet file is a special text file, which still stores data in ASCII format. The format file has been made some special requirements in order that excel and other spreadsheet processing software can directly read the data file. The output of spreadsheet file is achieved by reading spreadsheet file, after the path of reading file and the column number of reading data are appointed, the VI can read data in the spreadsheet file. The bottom layer 
functions such as read file can also be used to achieve output of spreadsheet file. The program block diagram is shown in Fig. 7. The informations of vehicles are stored in spreadsheet files which can save the randomly generated vehicle information at every runtime, so that can be used in debugging and editing of vehicle monitoring system. At the same time the vehicle informations are stored in the specified location file which can be refered later.

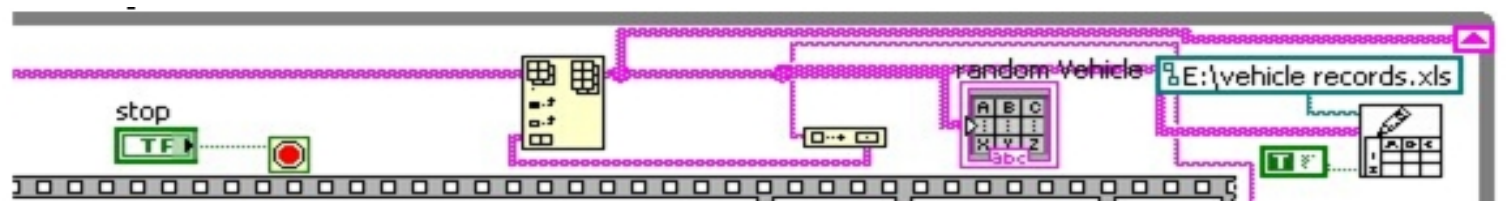

Fig. 7 Data storage and display module

Overall application process.Because the vehicle on the highway is random and sudden, the final signal simulation program is composed of random number, case structure and sequence structure. So the continuity and randomness of the signal are achieved, and the vehicle signal on the highway can be accurately simulated. The numbers of axes are determined through the size of random data. The jump is achieved through the case structure. The vehicle information is finally output, which is displayed on the front panel, and the vehicle information is recorded in the spreadsheet. The whole program block diagram is shown in Fig. 8.

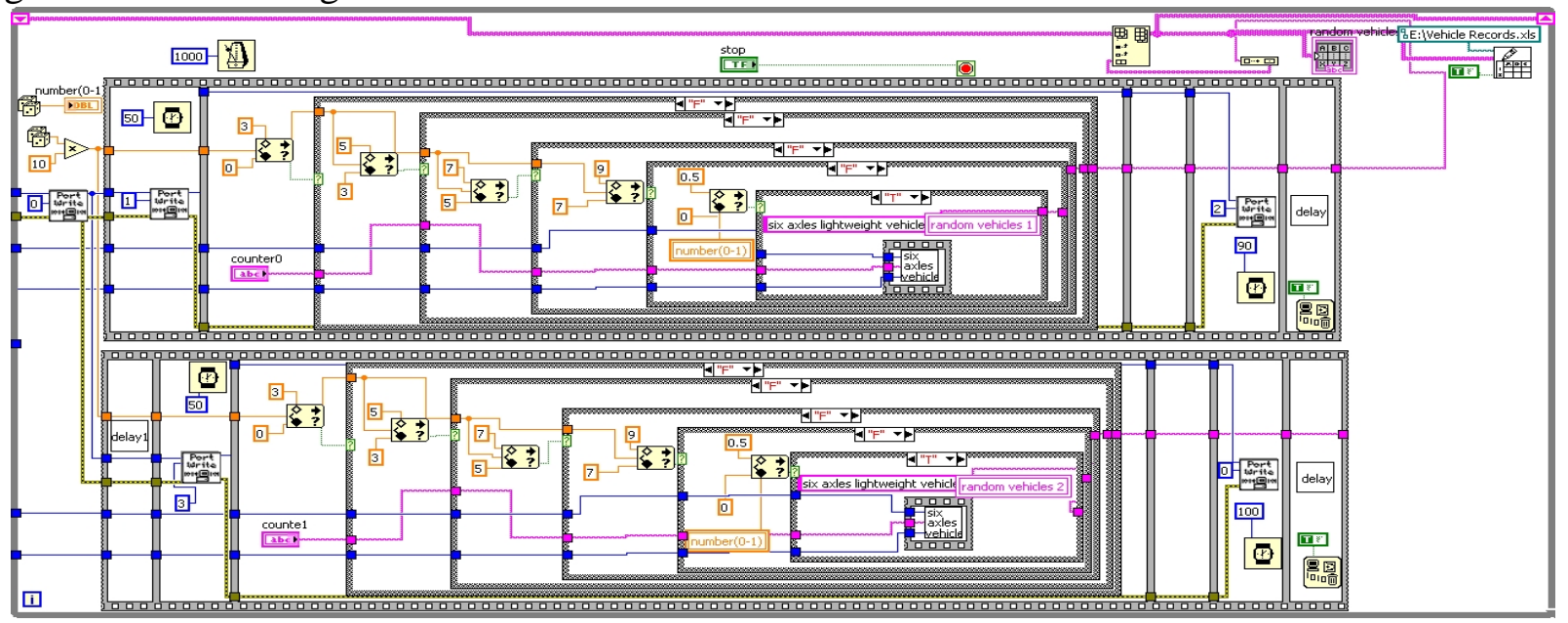

Fig. 8 Whole program block diagram

The program execution principle of vehicle signal simulation is as follows: the digital output ports output low level at the same time which is used for the simulation of initial signal of geomagnetic coil. That expresses that the vehicles entry into the detection zone. Then the random number generators generate random numbers. The numbers of axles are determined by the size of random numbers: the output is two axles vehicle when the numbers are between 0 to 3 , the output is three axles vehicle when the numbers are between 3 to 5, the output is four axles vehicle when the numbers are between 5 to 7 , the output is five axles vehicle when the numbers are between 7 to 9 , the output is six axles vehicle when the numbers are between 9 to 10 . After the random numbers are judged by the judgment program the program jumps to the program block diagram of the vehicle signals that need to output. Meanwhile, the random number generators generate random numbers again, which are used to determine the difference of the vehicles weight. Then the vehicle information is output. And the digital port output high level which simulates signal of geomagnetic coil. It shows that the vehicle leaves detection area. When the simulation signals of piezoelectric sensor are outputted the simulation signals of two piezoelectric sensors have slightly delay, so that they can match with the field collecting signal of piezoelectric sensor. When the vehicle signal output is completed, the digital signal output is changed to high level. It shows that the simulation signal of the geomagnetic coil is end. Then add random delay, which is used for the randomness of the vehicle on the highway. Thus it can accurately simulate the vehicle signals. Finally, release the memory unit by the application of memory release function, and reduce usage rate of memory. The vehicle signals are collected and displayed. 
Vehicle signal simulation results of system operation. The simulation signal of two axles vehicle is shown in Fig. 9. In Fig. 9 the curve 1 represents simulation signal of piezoelectric sensor 1, the curve 2 represents simulation signal of piezoelectric sensor 2, the curve 3 represents simulated signal of geomagnetic coil 1 , the curve 4 represents simulated signal of geomagnetic coil 2 . The signal buffer is set to 5000, the acquisition points at every time are 400, the channels of counter 0 and counter 1 are simulation signal of piezoelectric sensor. The digital channels 0 and 1 are the simulation signal of geomagnetic coil. From the results we can see that two piezoelectric sensors signal the same, but there is a certain delay; two geomagnetic coils signals are the same, and also there is a certain delay, the delay time are the same to piezoelectric sensor's. Data storage module displays the vehicles types, and the random vehicles are stored in the list, which is convenient for analyzing the vehicle signals in the vehicle monitoring system. The control panel contains the setup of buffer size, acquisition points and collection channels.

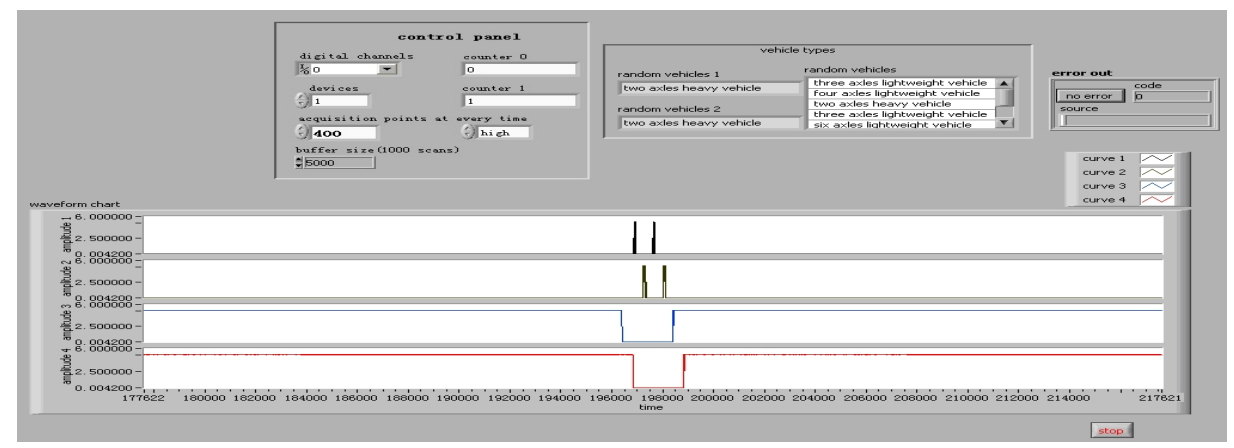

Fig. 9 Simulation signal of two axles vehicle

Comparison of simulated signals and field signal.By the acquisition of field signals to realize the contrast of field signal and simulation field. The field signal of two axles vehicle is shown in Fig.10.

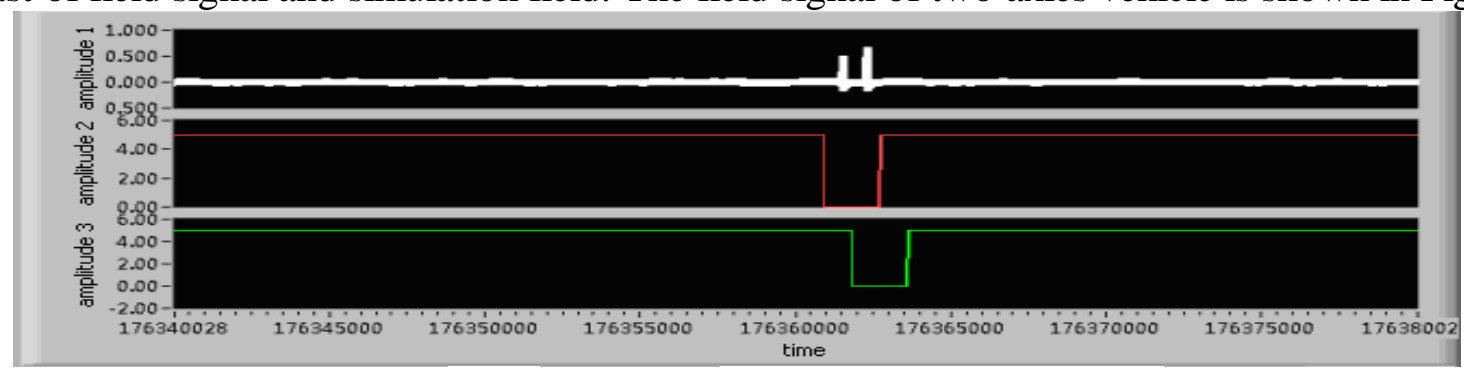

Fig. 10 Field acquisition signal of two axles vehicle

From Fig. 10 we can see: the first curve is the piezoelectric sensor signal, which displays the change of voltage, the second and third curves are the signals of geomagnetic coil 1 and 2 . When the vehicle drive into the detection zone the geomagnetic coils first detects the arrival of the vehicle, then the geomagnetic coil outputs low level, it means that the vehicle drives into the detection area. The piezoelectric sensor collects the vehicle information and records the vehicle peak information and peak time etc. When geomagnetic coil 2 output rising edge it means that the vehicle drives out the detection area.From the contrast of field acquisition signal and simulation signal we can see that the simulation signal can simulate field acquisition signal, the specific parameter that the vehicle axle load monitoring system need can be get from the simulation signal, so that axle load measurement is proceeding.

\section{Conclusions}

The timer port of PCI-6013 outputs pulse which simulates the piezoelectric sensor signal. The digital port of PCI-6013 outputs pulse which simulates geomagnetic coil signal. The above two simulation signals can form the vehicle signal. The randomness of the vehicles on the highway is simulated by the use of random data. And at last the simulation of the vehicle signal has been achieved. 


\section{Acknowledgements}

This work was financially supported by the Youth Foundation Project of Hebei Province Education Department (Q2012118).

\section{References}

[1] J.F. Wang, M.G. Wu: Proc. World Congr. Intelligent Control Autom. Vol. 6(2004), p. 5241

[2] L. Cheng, H.J.Zhang and X.H.Cao: Chin. J. Sci. Instrum. Vol. 27 (2006), p. 943

[3] Z.F. Zhou, P. Cai and R.X. Chen: IET Sci. Meas. Technol. Vol. 4(2007), p. 185

[4] G. Yannis: ITE J. Vol. 75(2005), p. 39

[5] Y. Ma, B.P.Chen and X.Wang: Traffic Eng. Technol. Nat. Defence No.3(2007), p. 35

[6] E.T. Yao, J. Ji and M. Zang: Transducer Microsyst. Technol. No.12(2005), p. 22 